\title{
MONITORAMENTO PARTICIPATIVO DA BIODIVERSIDADE EM SISTEMAS DE UNIDADES DE CONSERVAÇÃO: 0 CASO DO PROBUC NO ESTADO DO AMAZONAS
}

\author{
Daniel Carneiro Costa (D) $\triangle$
}

Universidade Federal do Amazonas I Manaus - AM - Brasil

Guillaume Antoine Emile Louis Marchand (D) $\triangle$

Universidade Federal do Amazonas I Manaus - AM - Brasil

Henrique dos Santos Pereira (1D $ه$

Universidade Federal do Amazonas I Manaus - AM - Brasil 


\section{RESUMO}

Nas últimas décadas, o monitoramento participativo tem sido apontado como um instrumento crescentemente importante para a consolidação da gestão de áreas protegidas. No estado do Amazonas, a criação, em 2005, do Programa de Monitoramento da Biodiversidade e do Uso de Recursos Naturais de Unidades de Conservação (ProBUC) representou um importante passo para a efetividade do monitoramento participativo das unidades estaduais de conservação (UC estaduais). Apresentamos, sinteticamente, os resultados de uma avaliação qualitativa do funcionamento deste programa, identificando suas contribuições e limitações para a vida das comunidades locais. Iniciamos com uma revisão da literatura sobre as principais razões do surgimento dos programas de monitoramento participativo e chegamos às observações dos atores sociais gestores, especialistas e comunitários, envolvidos na construção do ProBUC, por meio de entrevistas. Constatamos que o programa contribuiu para a conscientização ambiental e produziu dados sobre a biodiversidade, embora não se tenha verificado o subsídio à gestão das unidades de conservação.

Palavras-chave: monitoramento participativo; gestão da biodiversidade; unidades de conservação.

\section{PARTICIPATORY MONITORING OF BIODIVERSITY IN CONSERVATION UNIT SYSTEMS: THE CASE OF PROBUC IN THE STATE OF AMAZONAS}

In the last decades, participatory monitoring has been pointed out as an increasingly important instrument for consolidating the management of protected areas. In the state of Amazonas, the creation of the Program for Monitoring the Biodiversity and the Use of Natural Resources (ProBUC), in 2005, represented an important step towards the effectiveness of participatory monitoring of state conservation units (state CUs). We briefly present the results of a qualitative evaluation of the functioning of this program, identifying its contributions and limitations to the life of local communities. We started with a literature review on the main reasons for the emergence of the participatory monitoring programs and we came to the observations of managers, specialists and community members social actors involved in the construction of ProBUC, through interviews. We found that the program contributed to environmental awareness and produced data on biodiversity, although the subsidy for the management of conservation units was not verified.

Keywords: participatory monitoring; management of biodiversity; conservation units.

\section{MONITOREO PARTICIPATIVO DE LA BIODIVERSIDAD EN SISTEMAS DE UNIDADES DE CONSERVACIÓN: EL CASO DEL PROBUC EN EL ESTADO DEL AMAZONAS}

En las últimas décadas, el monitoreo participativo ha sido señalado comouninstrumentocrecientementeimportanteparalaconsolidación delagestióndeáreas protegidas.EnelEstadodeAmazonas,lacreación, en 2005, delPrograma de Monitoreo de la Biodiversidad y Uso de los Recursos Naturales - ProBUC - representó un importante paso para la efectividad del monitoreo participativo de las unidades estatales de conservación (UCs estaduales). Presentamos, sintéticamente, los resultados de una evaluación cualitativa del funcionamiento de este programa, identificando sus contribuciones y limitaciones a la vida delas comunidades locales. Iniciamos con una revisión delaliteratura sobre las principales razones del surgimiento de los programas de monitoreo participativo y llegamos a las observaciones de los actores sociales gestores, especialistas y comunitarios involucrados en la construcción delProBUC, a través de entrevistas. Coonstatamos queel programa contribuyóa la concientización ambiental y produjo datos sobre la biodiversidad, aunque no se constató el subsidio a la gestión de las unidades de conservación.

Palabras clave: monitoreo participativo; gestión de la biodiversidad; unidades de conservación. 


\section{INTRODUÇÃO}

Em termos gerais, os programas de monitoramento de áreas protegidas foram criados com o propósito de auxiliar na gestão dessas áreas, por meio da produção de dados para identificar tendências da biodiversidade (Boissière et al. 2014:150; Lee et al. 2005:3; Schmeller et al. 2009:308). Os programas de monitoramento participativo, de modo mais particular, foram concebidos como propulsores da participação de comunidades usuárias da biodiversidade no processo de gestão (Danielsen et al. 2000:1672). Como observaram Magnusson et al. (2013:21), eles são cada vez mais utilizados na promoção da gestão participativa da biodiversidade, inclusive nas áreas protegidas brasileiras denominadas de unidades de conservação (UC).

Contudo, para se chegar a esse patamar de reconhecimento e de valorização do monitoramento participativo, é importante salientar que o feito de forma convencional, exclusivo dos cientistas, revelou-se insuficiente para auxiliar na gestão da conservação da biodiversidade. Isso resultou na criação da estratégia de envolvimento das comunidades usuárias da biodiversidade no processo de gestão e de monitoramento, implicando uma "coordenação social”, que significa a aprendizagem decorrente de um processo de gestão com distintos atores relacionados, como pesquisadores, gestores públicos e comunidades (Danielsen et al. 2010:3; Stuart-Hill 2005:2622; Cundill \& Fabricius 2009:3206).

Este processo ganhou visibilidade na década de 1980, quando as agências internacionais de financiamento para criação e consolidação de áreas protegidas passaram a exigir dos governos a elaboração de projetos que alcançassem a participação direta de comunidades na própria gestão das áreas protegidas (Estrella \& Gaventa 1998:3). Essa nova metodologia de monitoramento passou a depender de aprovações dentro das organizações das comunidades locais (Garcia \& Lescuyer 2008:1304) e favoreceu, em alguns países, a aceleração de inventários biológicos e as tomadas de decisões, que evitaram a extinção de determinadas espécies biológicas (Sheil \& Lawrence 2004:634).

É neste cenário que a criação do Programa de Monitoramento da Biodiversidade e do Uso de Recursos Naturais de Unidades de Conservação (ProBUC) se insere no contexto de reconhecimento da relevância do monitoramento participativo no processo de consolidação de áreas protegidas no estado do Amazonas.

Este programa foi concebido para monitorar as dinâmicas das espécies amazônicas e também para viabilizar o uso sustentável dos recursos naturais. Seus objetivos principais eram

gerar, continuamente e de forma
participativa, informações estratégicas
para a gestão de unidades de
conservação [...] e permitir a inserção
das comunidades no processo decisório
das ações de gestão da UC onde estão
localizadas (Fonseca Junior et al. 2011:15).

De um lado, acreditava-se que o ProBUC seria capaz de identificar estados críticos relacionados à sobrevivência de certas espécies, a fim de orientar a gestão das unidades de conservação e de conscientizar os próprios comunitários sobre a necessidade de reduzir ou minimizar o uso da biodiversidade pressionada. Por outro, esperava-se que esse monitoramento facilitasse a avaliação da 
disponibilidade de espécies passíveis de utilização planejada, inclusive para fins econômicos, como no caso das atividades de manejo extrativo.

A pesquisa efetuada demonstrou que o programa apresenta lacunas para cumprir adequadamente seus objetivos previstos, o que exige ajustes estruturais na sua gestão, para que não tenha problemas de continuidade. Todavia, verificou-se que ele colaborou para reforçar a conscientização da proteção da biodiversidade amazônica, por meio do protagonismo das comunidades existentes no interior das unidades de conservação.

\section{PROCEDIMENTOS METODOLÓGICOS}

\subsection{PARTICULARIDADES DO PROBUC: ÁREAS DE ABRANGÊNCIA, GESTÃO E OBJETOS DE MONITORAMENTO}

De acordo com Costa (2014:17), o programa foi implantado, em 2005, na Reserva de Desenvolvimento Sustentável (RDS) do Uacari, localizada no município de Carauari. Em 2007, o programa foi estendido ao Parque Estadual (PAREST) do Rio Negro Setor Norte, no município de Novo Airão; e, em 2009, ocorreu sua terceira implantação, dessa vez, na RDS Uatumã, entre os municípios de São Sebastião do Uatumã e de Presidente Figueiredo. É importante observar que, na fase piloto, ele foi implantado em duas UC de uso sustentável (áreas que contemplam a convivência humana) e em uma de proteção integral (áreas com restrições para a presença e para atividades humanas).

Em 2018, conforme informações obtidas junto ao Departamento de Mudanças Climáticas e Gestão de Unidades de Conservação (DEMUC), vinculado à Secretaria de Estado do Meio Ambiente do Estado do Amazonas (SEMA), o programa encontra-se implementado nas seguintes unidades de conservação estaduais: RDS Puranga Conquista (área rural de Manaus); RDS Madeira (abrangendo parte dos municípios de Novo Aripuanã e Manicoré); RDS Amapá (também nas proximidades do município de Manicoré); RDS Matupiri (nos limites dos municípios de Borba e Manicoré); Reserva Extrativista (RESEX) Canutama (localizada no município de mesmo nome); PAREST Rio Negro Setor Sul (o mesmo acesso do PAREST Rio Negro Setor Norte); PAREST Matupiri; e Mosaico do Apuí (no município de Apuí).

No ProBUC, as espécies e os bens comuns que são objetos de monitoramento são chamados "componentes". Até 2014, quando da consolidação da pesquisa de dissertação de mestrado intitulada "Limites e potencialidades do Programa de Monitoramento da Biodiversidade e do Uso de Recursos Naturais - ProBUC - para a gestão ambiental de unidades de conservação do Amazonas", no âmbito do Programa de Pós-Graduação em Ciências do Ambiente e Sustentabilidade na Amazônia, da Universidade Federal do Amazonas (PPGASA-UFAM), os seguintes componentes haviam sido aprovados para implementação paulatina: medição do tamanho das espécies e estimativa da quantidade de quelônios e de jacarés; recenseamento das atividades produtivas e do consumo dos comunitários entrevistados; especificação do trânsito de embarcações, mediante anotação dos barcos que ingressam nas áreas de interesse, com horários específicos; observação em trilha pré-definida dos animais silvestres, por meio de anotação e do registro da captura 
de pescado ${ }^{1}$ (Costa 2014:17). O programa em análise custou mais de R 1 milhão, distribuídos entre 2006 e 2011, ficando 9\% acima do que fora projetado. Recentemente, soubemos que apenas três componentes prosseguem funcionando: o de quelônios, o de trânsito de embarcações e o de recenseamento.

A gestão do ProBUC esteve, originalmente, sob responsabilidade do Centro Estadual de Unidades de Conservação (CEUC), órgão da Secretaria de Desenvolvimento Sustentável (SDS), atualmente denominada de Secretaria de Estado do Meio Ambiente (SEMA). Por conseguinte, o CEUC passou a ser chamado de Departamento de Mudanças Climáticas e gestão de UC (DEMUC), tendo sua estrutura reduzida, em comparação com a anterior. Havia, de início, o objetivo de estender o ProBUC ao conjunto das UC estaduais, integrantes do Sistema Estadual de Unidades de Conservação (SEUC), que, por seu turno, compõe o Sistema Nacional de Unidades de Conservação (SNUC), principal arranjo institucional das áreas protegidas brasileiras.

Em tese, o ProBUC deveria alimentar, com dados obtidos da monitoria comunitária, o próprio órgão ambiental estadual, qualificando e integrando a gestão das UC. Este era um propósito bastante ambicioso, haja vista as dificuldades inerentes às dimensões continentais do estado do Amazonas.

\subsection{OBJETIVOS DA PESQUISA E INSTRUMENTOS METODOLÓGICOS}

A pesquisa empreendida procurou avaliar qualitativamente o funcionamento do ProBUC a partir da contribuição dos grupos de interesse diretamente relacionados: gestores, pesquisadores e comunitários. Procurou-se obter um diagnóstico do programa, com foco principal nas opiniões destes atores sobre as deficiências e os potenciais do programa. Para tanto, os instrumentos metodológicos empregados foram: pesquisa bibliográfica e documental, entrevistas semiestruturadas e observação direta.

A pesquisa bibliográfica consistiu no debate teórico sobre o tema do monitoramento participativo da biodiversidade, abrangendo seu histórico e os seus fundamentos. Por sua vez, a pesquisa documental representou o acesso a informações oficiais sobre o programa que estavam em publicações do CEUC.

As entrevistas semiestruturadas obedeceram a um roteiro pré-definido, que procurava extrair dos entrevistados o máximo de informações e de impressões sobre o programa. Estas entrevistas foram realizadas entre outubro de 2013 e junho de 2014.

As entrevistas com cinco pesquisadores do ProBUC transcorreram em Manaus, entre outubro e dezembro de 2013. Entre os critérios escolhidos, houve os da participação na implementação ou na avaliação do programa, além da utilização de dados contidos no CEUC para publicações acadêmicas.

As entrevistas com seis gestores envolvidos com o programa distribuíram-se em dois momentos: na sede do CEUC, ainda em 2013, quando houve encontros com três servidores responsáveis diretamente pela gestão do ProBUC, e nas viagens às unidades de conservação (RDS Uatumã, RDS Uacari e PAREST Rio Negro Setor Norte), ocasião

1 Esse componente não foi implementado nas UC visitadas durante a pesquisa de dissertação de mestrado, nem naquelas em que o programa foi, posteriormente, efetivado. 
em que os três gestores de cada uma dessas UC foram entrevistados.

Nestas mesmas viagens às UC, efetivadas entre fevereiro e junho de 2014, cerca de 67 comunitários foram entrevistados. Ao todo, foram 30 na RDS Uatumã, 27 na RDS Uacari e dez no PAREST Rio Negro Setor Norte 2 . Como critérios, procurou-se entrevistar comunitários que participavam do programa como monitores, entrevistados de recenseamento, lideranças vinculadas ao conselho deliberativo da UC e até mesmo comunitários que não tinham participação permanente no ProBUC, mas que eventualmente estiveram em alguma reunião que abordou o programa. Este esforço resultou na totalidade de 42 monitores e de 25 não monitores entrevistados.

Por meio das respostas dadas nestas entrevistas pelo conjunto dos grupos de interesse, identificaram-se os principais aspectos favoráveis e desfavoráveis da integração do programa à vida das comunidades em que foi estabelecido, debatidos à luz da literatura disponível. Este panorama permitiu a apresentação de um conjunto de recomendações que visava colaborar com o aprimoramento da gestão do programa.

A avaliação exposta neste trabalho é uma síntese dos resultados da dissertação de mestrado mencionada (Costa 2014), que se destinou a conhecer as principais limitações e deficiências do ProBUC, objetivando propor alterações em seu funcionamento, para melhorar seu desempenho.

\section{RESULTADOS DO PROBUC À LUZ DOS DESAFIOS GLOBAIS DO MONITORAMENTO PARTICIPATIVO}

\subsection{PONDERAÇÕES SOBRE O PROBUC BASEADAS EM DEPOIMENTOS DE GESTORES E DE ESPECIALISTAS}

Entre os aspectos essenciais e críticos do ProBUC ressaltados pelos especialistas e gestores, elencamos os limites da participação comunitária, o financiamento e o retorno de dados. Em linhas gerais, esses dois atores sociais defenderam o caráter participativo do programa. Contudo, as entrevistas realizadas com os gestores do CEUC nos revelaram que, diante dos diversos níveis de envolvimentos dos comunitários com as iniciativas de conservação da biodiversidade, fez-se importante apostar na aprovação de perfis de monitores que se adaptassem melhor à proposta de funcionamento do programa.

Soubemos que as indicações da equipe técnica do ProBUC passaram pelo aval da maioria dos moradores presentes nas reuniões comunitárias que se destinavam a aprovar a implementação do programa. Para tanto, os técnicos partiram da identificação de potenciais monitores nos primeiros contatos feitos com os comunitários, a fim de convencê-los a aderir ao programa. Essa identificação prévia foi importante para a inclusão de pessoas com afinidades em cada uma das funções de monitoria - algo que seria aprovado apenas posteriormente pelos

2 A realização das entrevistas em campo foi possível graças ao financiamento da Fundação de Amparo à Pesquisa do Estado do Amazonas (FAPEAM), por meio do projeto "Sustentabilidade socioeconômica e ambiental do programa de monitoramento da biodiversidade e do uso de recursos naturais em unidades de conservação do Amazonas". Esse financiamento garantiu a logística necessária para os deslocamentos até as unidades de conservação onde vigorava o programa. A pesquisa foi registrada no Comitê de Ética sob o número 23250113.2.0000.5020. 
comunitários. Nesse sentido, pode-se afirmar que houve influência técnica na indicação dos monitores, sob a alegação da relevância da escolha de pessoas adequadas às necessidades de cada trabalho para que o programa obtivesse resultados consistentes.

Por sua vez, os especialistas alegaram que não houve divergências significativas quanto aos procedimentos adotados pelos técnicos do programa para incluir a participação comunitária. Para eles, tal como para os gestores, efetivou-se o cumprimento das formalidades que conferiram caráter participativo ao processo, o que garantiu a incorporação dos interesses comunitários tanto para a escolha dos monitores como para a seleção dos componentes.

O cumprimento formal da participação comunitária em um programa de monitoramento, todavia, não é suficiente para garantir que os interesses de todas as partes envolvidas estejam corretamente atendidos. A esse respeito, Evans \& Guariguata (2008:10) apontam a existência de uma divisão de grupos de interesse na construção desse tipo de programa: de um lado, estariam as populações locais, que detêm uma forma direta de uso dos recursos ou das espécies, em conformidade com seu modo de vida; de outro, os pesquisadores, os governos e as organizações não governamentais, geralmente imbuídos de interesses protecionistas, mais do que o primeiro grupo de interesse. Esse segundo grupo traz recursos financeiros e técnicos essenciais para a institucionalização dos programas; já os usuários da biodiversidade são essenciais para a constituição de um monitoramento efetivamente democrático.

Por essa razão, a negociação permanente de prioridades provenientes dos grupos de interesse é fundamental para que os programas funcionem com legitimidade (Lawrence et al. 2007:329). No caso do ProBUC, não tivemos condições de avaliar com maior profundidade se as negociações que resultaram nos componentes aprovados também pelos comunitários refletiram, da maneira mais fidedigna possível, os interesses dessas populações locais. No máximo, como mencionado, soubemos que os componentes aprovados não encontraram objeções das comunidades, assim como sua indicação foi baseada em particularidades apontadas pelos planos de manejo das unidades de conservação em que o programa se efetivaria.

Em relação ao financiamento do programa, gestores e especialistas confirmaram a dependência que o ProBUC mantinha em relação a repasses da fundação norte-americana Moore, o que corroborou a deficiência crônica consistente no vínculo que programas de monitoramento participativo de diversas partes do mundo possuem no que concerne a recursos externos (Whitelaw et al. 2003:412). Essa situação é mais crítica quando se trata de programas situados em países periféricos ou em desenvolvimento, onde a limitação orçamentária acentuada e permanente torna o funcionamento deles mais dependente de agências internacionais e de organizações não governamentais (Danielsen et al. 2007:566).

O ProBUC não fugia à regra dos programas com dependência financeira externa e que sofrem com a instabilidade dessa condição. Tanto gestores quanto especialistas reconheceram que essa dependência tinha um reflexo direto na rotina das atividades. As renovações contratuais eram períodos em que o programa ficava paralisado, sem recursos, com sérios problemas de continuidade. Associado a 
isso, havia elevada rotatividade da equipe técnica. Normalmente, os técnicos ocupavam cargos comissionados, com permanência incerta, pois a mudança na direção da secretaria de meio ambiente significava alteração também desses membros do ProBUC. E tais alterações, muitas vezes bruscas, implicavam a dissolução de laços de confiança e de afetividade criados entre a equipe técnica e os próprios comunitários, que acabavam se desanimando com sua participação no ProBUC, além de serem obrigados a aguardar o retorno das atividades por tempo incerto, como testemunhado em algumas entrevistas.

Como terceira limitação relevante destacada pelos gestores e especialistas entrevistados, devemos assinalar que o ProBUC não cumpriu a principal tarefa de oferecer dados que auxiliem na gestão das áreas protegidas. No entanto, gestores e especialistas defenderam que o programa, na prática, contribuiu para valorizar conhecimentos tradicionais por meio da monitoria, discutindo a importância da conservação e recolhendo dados para a gerência do programa, além de ter subsidiado pesquisas acadêmicas.

Como ressaltado pelos próprios gestores, os dados foram expostos em reuniões comunitárias, para que fossem socializados ao máximo entre as pessoas locais. Também ficaram disponíveis em um banco de informações localizado no CEUC, sendo utilizados por pesquisadores para projetos específicos, mediante formalização. Mas essas informações não serviram como base para a elaboração de políticas que pudessem aperfeiçoar a gestão das unidades de conservação, como para a criação de planos de manejo e para a revisão de zonas de proteção da biodiversidade, o que corresponderia à razão primordial desta iniciativa. Nesse sentido, os especialistas foram mais críticos ao afirmarem que faltou do próprio governo estadual uma política ambiental que conferisse ao monitoramento participativo uma função estratégica para alimentar e subsidiar a gestão das unidades de conservação, através do uso sistemático dos dados apurados pela monitoria comunitária.

Essa problemática do retorno de dados como benefícios não é, certamente, específica do ProBUC, pois Garcia \& Lescuyer (2008:1304) utilizam a ideia de "relação disfuncional" para descrever o desencontro entre a produção participativa de dados e o aproveitamento deles na gestão da área protegida. É necessário enfatizar, do mesmo modo, que também há exemplos de aproveitamento do retorno dos dados coletados pelos programas de monitoramento, por intermédio de políticas públicas que ajustaram regras de uso e legislações sobre a biodiversidade monitorada, como as experiências verificadas nos Estados Unidos e nas Filipinas (Andrianadransana et al. 2005:2760; Danielsen et al. 2007:569).

\subsection{A CONSISTÊNCIA DO PROBUC NA PERSPECTIVA DOS COMUNITÁRIOS}

Nas entrevistas realizadas com os comunitários, entre os principais pontos positivos sobre a existência do ProBUC, foram destacadas a melhoria do conhecimento sobre biodiversidade, os recursos naturais e o seu uso (19,5\%) e a ajuda financeira oferecida aos monitores $(15,9 \%)$. A primeira vantagem demonstra o reforço ambiental representado pelo programa. Os moradores locais informaram que, desde a criação da área protegida, 
são comuns cursos e oficinas que trabalham a perspectiva da conscientização ambiental. Assim, o ProBUC aparece como mais uma oportunidade para que eles conheçam a importância do uso controlado de espécies e de recursos naturais no interior da UC em que vivem.

A esse respeito, é interessante sublinhar que Garcia \& Lescuyer (2008:2) demarcam que a metodologia participativa presume uma aproximação entre comunidades e estruturas do poder estatal, na tentativa de descentralizar a tomada de decisões na "gestão sustentável". Ela funciona como um ensejo para que os próprios usuários da biodiversidade, com base no conhecimento produzido e difundido pelo monitoramento, revejam suas práticas, com o fito de adaptá-las a condições de menor impacto.

Essa sensibilização ambiental comunitária, contraditoriamente, não deixa de ser impositiva, porque "subjuga [as] práticas locais às novas restrições ecológicas" (Andrianadransana et al. 2005:2758). Por essa razão, a criação de regras para o uso da biodiversidade precisa estar vinculada às organizações comunitárias. Para Ghate \& Nagendra (2005:511), ações coletivas voltadas para a regulação do acesso e da utilização da biodiversidade devem ser frutos de um processo de convencimento, fundamentado nas regras definidas pelos próprios comunitários usuários, não em ideias simplesmente impostas por agentes externos.

Quanto ao segundo ponto positivo do ProBUC, cabe frisar que, entre as razões para aceitar a monitoria, as duas principais respostas dividiramse entre vontade de conhecer melhor a reserva e seus recursos naturais $(21,8 \%)$ e sensibilidade para valorizar os recursos naturais (14,5\%). Como foi uma seleção de voluntários, no ato da candidatura, os comunitários realmente não sabiam se haveria ajuda de custo, algo que lhes foi revelado apenas após os resultados da aprovação, durante a oficina de capacitação. No momento desta pesquisa, a ajuda de custo, equivalente a diárias de trabalho, consistia nos seguintes valores: $\mathrm{R} \$ 25,00$ por dia de monitoramento, sendo que os de fauna e de jacaré, por serem considerados os de maior periculosidade, chegavam a $\mathrm{R} \$ 35,00$. Como, em média, a atividade do monitoramento ocupava os colaboradores uma vez a cada quinzena, eles chegavam a receber, de modo bimestral, entre $\mathrm{R} \$$ 100,00 e $\mathrm{R} \$ 140,00$ por este trabalho. Ademais, na RDS Uacari, os monitores de quelônios recebiam um rancho mensal referente a $\mathrm{R} \$ 300,00$. Estes valores foram considerados importantes, uma vez que a renda mensal da população era baixa, sendo, em média, de $\mathrm{R} \$ 770,91$ por domicílio. No entanto, a retribuição financeira foi avaliada como insuficiente por alguns dos monitores $(17,20 \%)$. Os riscos oferecidos e o tempo empregado para a monitoria apareceram como motivos para justificar a necessidade de melhorias no valor concedido. Justamente em decorrência da dedicação ao programa, cerca de $50 \%$ dos monitores entrevistados afirmaram que não executariam mais o trabalho sem recompensa financeira.

Entretanto, algumas pessoas declararam que, mesmo sem qualquer remuneração, permaneceriam na função, pela satisfação de conhecer a biodiversidade $(18,2 \%)$ e por considerála um trabalho útil à comunidade $(27,30 \%)$. Deve ser feita menção especial aos colaboradores que já realizavam o monitoramento de quelônios antes 
da chegada do ProBUC. Uma expressiva parcela de monitores da RDS Uacari $(62,5 \%)$ garantiu que continuaria o monitoramento sem remuneração. Muitos acreditavam que esse trabalho aumentaria a quantidade de quelônios, ainda que não dispusessem de dados comprobatórios, até o momento da investigação.

Sobre esse tema, Danielsen et al. (2010:1167) comentam que o pagamento pode colaborar para que haja mudanças nas atitudes dos comunitários, que passariam a adotar comportamentos mais conservacionistas em relação aos recursos da biodiversidade. Por seu turno, Jorgensen et al. (2005:2669) alegam que o comprometimento com o dever do monitoramento precisa ser compensado por incentivos econômicos, uma vez que tal atividade ocupa um tempo que poderia ser gasto pela população em suas tarefas cotidianas, relacionadas especialmente à sobrevivência. Contudo, os autores enfatizam que a compensação se justifica dentro da estratégia de incentivos sociais, em benefício de toda a comunidade.

Dando prosseguimento aos resultados obtidos, as principais deficiências apresentadas pelos comunitários sobre o ProBUC relacionamse à existência de dificuldades para definir o programa $(16,5 \%)$ e ao desconhecimento sobre a utilidade dos dados produzidos por ele (34\%). Verificamos mais comunitários não monitores que desconheciam o programa (21\%) do que comunitários monitores que o desconhecessem (13,5\%). De todo modo, é preocupante a constatação de que havia participantes diretos que não sabiam defini-lo. Afinal, todos os monitores receberam formação para esse tipo de trabalho, incluindo, é claro, informações básicas sobre os objetivos e as razões do programa.

É possível que essa formação sobre o programa, realizada pelos técnicos, tenha sido insuficiente para conduzir a uma compreensão mais abrangente dele. Contudo, a articulação entre conhecimentos práticos das comunidades locais e o conhecimento científico propicia a valorização da experiência de moradores que são usuários diretos da biodiversidade, enriquecendo a gestão da biodiversidade (Reed et al. 2008:1253; Evans \& Guariguata 2008:5).

Em referência ao desconhecimento dos dados produzidos pelo monitoramento, uma parcela expressiva dos entrevistados não monitores informou nunca ter tido nenhum contato com os resultados do ProBUC (65,5\%). É um nível de desinformação bastante elevado, o que certamente comprometeu o engajamento comunitário, haja vista a dificuldade de se obter apoio da população para algo que é por ela desconhecido. Entretanto, a maioria dos monitores (63\%) alegou que os resultados eram divulgados nas reuniões comunitárias. Pelo percentual observado, esse procedimento parece ter sido insuficiente para popularizar os resultados do programa.

Destacamos, ainda, que existiam dificuldades de compreensão dos resultados até mesmo por parte dos monitores (23\%), os quais disseram, na ocasião, entender "parcialmente" os boletins encaminhados pela equipe técnica do programa. Ademais, alguns deles (13\%) declararam simplesmente que não compreendiam nenhum dos resultados expressos nos boletins. Considerando isso, é possível afirmar que as incompreensões sobre os dados e as informações por parte dos próprios monitores 
prejudicavam a divulgação deles aos demais comunitários.

Essa questão nos remeteu ao problema mais amplo de comunicação dos resultados do ProBUC. Para ultrapassar esse obstáculo, há a necessidade de criar alternativas que facilitem a compreensão dos dados pelos comunitários. A respeito disso, Guijit (2008:36) salienta que, muitas vezes, os programas de monitoramento acabam encerrando-se no papel de meros produtores de dados, sem materializar um retorno sistemático, condizente com as condições sociais de aprendizagem, que seria fundamental para nivelar um entendimento mínimo e comum para todos os atores que participam do processo.

Mesmo desconhecendo ou não sabendo utilizar os dados produzidos, em geral, os comunitários acreditavam que o ProBUC poderia contribuir para melhorias em suas estratégias produtivas. Aproximadamente $25 \%$ imaginavam ou desejavam que o programa ajudasse na geração de renda e no aumento de oportunidades econômicas. $\mathrm{Ou}$ seja, o envolvimento passava pela expectativa de satisfação de suas necessidades. Magnusson et al. (2013:21) assinalam que a participação direta dos comunitários no monitoramento participativo se torna viável à medida que os programas se vinculam a direitos socioeconômicos, como reconhecimento do uso da terra, do rio e da biodiversidade, geração de renda, entre outros benefícios.

Um exemplo notável, observado por Danielsen et al. (2007:569), ocorreu nas Filipinas, onde o monitoramento facilitou a racionalização do uso da biodiversidade pelos comunitários usuários, que atuaram como gestores dos próprios parques onde vivem com seus sistemas tradicionais. Gimenez et al. (2008:2) asseveram que os benefícios se associam também ao protagonismo comunitário em todo o processo de gestão do programa, em conjunto com as diferentes etapas, como aprovação de protocolos, realização de coleta de dados e análise dos resultados, produzindo, assim, o denominado "monitoramento colaborativo".

\section{CONSIDERACÕES FINAIS}

Um programa de monitoramento que pretenda integrar todas as gestões das UC estaduais do Amazonas é um desafio, e exige um maciço investimento. Nesse sentido, o ProBUC foi uma interessante iniciativa.

No contexto de consolidação das unidades de conservação estaduais, este programa contribuiu com a capacidade de mobilizar moradores locais em torno da proposta da monitoria comunitária, debatendo a importância da conservação da biodiversidade e gerando dados, que podem indicar tendências relevantes para subsidiar as intervenções públicas nas UC em que o programa foi inserido. Inegavelmente, serviu como suporte econômico para comunitários que dele participaram, embora isso não constasse como objetivo do programa.

A existência do ProBUC até os dias atuais, ainda que reduzido a poucos componentes, é uma demonstração de sua viabilidade, até porque está estendido a um número maior de UC. Fortalecê-lo é um imperativo. Para tanto, é fundamental que os recursos do programa federal "Áreas Protegidas da Amazônia”, que hoje o sustentam, sejam capazes de financiá-lo em longo prazo, permitindo investimentos em logística e na promoção de uma equipe técnica permanente, para que não haja mais problemas de continuidade. 
Da mesma forma, é inadiável que os dados acumulados ao longo da existência do programa sejam direcionados tanto para a revisão dos planos de gestão das UC quanto para os órgãos responsáveis pela implementação dos planos de manejo, gerando programas de renda que atendam e motivem as comunidades usuárias diretas da biodiversidade.

\section{REFERÊNCIAS}

Andrianadransana, H. T., J. Randriamahefasoa, J. Durbin, R. E. Lewis, e J. H. Ratsimbasafy, 2005. Participatory ecological monitoring of the Alaotra wetlands in Madagascar. Biodiversity \& Conservation 14(1):2757-2774. DOI: https://doi. org/10.1007/s10531-005-8413-y.

Boissière, M., F. Bastide, I. Basuki, J. L. Pfund, e A. Boucard. 2014. Can we make participatory NTFP monitoring work? Lessons learnt from the development of a multistakeholder system in Northern Laos. Biodiversity Conservation 23(1):149-170. DOI: https://doi.org/10.1007/s10531013-0589-y.

Costa, D. C. 2014. Limitações e potencialidades do Programa de Monitoramento da Biodiversidade e do Uso de Recursos Naturais - ProBUC - para a gestão ambiental de unidades de conservação do Amazonas. Dissertação de Mestrado, Departamento de Ciências do Ambiente e Sustentabilidade na Amazônia, Universidade Federal do Amazonas, Brasil.

Cundill, G., e C. Fabricius. 2009. Monitoring in adaptive co-management: toward a learning based approach. Fournal of Environmental Management 90(11):3205-3211. DOI: https://doi.org/10.1016/j. jenvman.2009.05.012.

Danielsen, F., D. S. Balete, M. K. Polsen, M. Enghoff, C. M. Nozawa, e A. E. Jensen. 2000. A simple system for monitoring biodiversity in protected areas of a developing country. Biodiversity \& Conservation 9(12):1671-1705. DOI: https://doi. org/10.1023/A:1026505324342.

Danielsen, F., M. M. Mendoza, A. Tagtag, P. A. Alviola, D. S. Balete, A. E. Jensen, M. Enghoff, e M. K. Polsen. 2007. Increasing conservation management action by involving local people in natura resource monitoring. Ambio 36(7):7:566-570. DOI: https://doi. org/10.1579/0044-7447(2007)36[566:icmabi]2.0.co;2.

Danielsen, F., N. D. Burgess, P. M. Jensen, e K. Pirhofer-Walzl. 2010. Environmental monitoring: the scale and speed of implementation varies according to the degree of peoples involvement. Journal of Applied Ecology 47(6):1166-1168. DOI: https://doi.org/10.1111/j.1365-2664.2010.01874.x.

Estrella, M., e J. Gaventa. 1998. Who counts reality? Participatory monitoring and evaluation: a literature review. (IDS working papers, 70 ). Brighton: Institute of DevelopmentStudies. Disponível em: https://opendocs. ids.ac.uk/opendocs/bitstream/handle/123456789/3388/ Wp70.pdf?sequence=1. Acesso em: 20 jul. 2016.

Evans, K., e M. R. Guariguata. 2008. Participatory monitoring in tropical forest management: a review of tools, concepts and lessons learned. Indonesia: Center for International Forestry Research. 
Disponível em: http://www.cifor.org/publications/ pdf_files/Books/BGuariguata0801.pdf. Acesso em: 8 out. 2016.

Fonseca Junior, S. F. et al. 2011. Programa de monitoramento da biodiversidade e do uso de recursos naturais - ProBUC: a experiência das unidades de conservação estaduais do Amazonas. Manaus: Centro Estadual de Unidades de Conservação do Amazonas.

Garcia, C. A., e G. Lescuyer. 2008. Monitoring, indicators and community based forest management in the tropics: pretexts or red herrings. Biodiversity \& Conservation 17(6):1303-1317. DOI: https://doi. org/10.1007/s10531-008-9347-y.

Ghate, R., e H. Nagendra. 2005. Role of monitoring in institutional performance: forest management in Maharashtra, India. Conservation \& Society 3(2):509-532. Disponível em: http://www.conservationandsociety.org/text. asp?2005/3/2/509/49325. Acesso em: 12 jul. 2016.

Gimenez, M. E. F., H. L. Ballardi, e V. E. Stutervant. 2008. Adaptive management and social learning in collaborative and community-based monitoring: a study of five community-based forestry organizations in the western USA. Ecology and Society 13(2):1-22. Disponível em: https://www.ecologyandsociety.org/ vol13/iss2/art4/main.html. Acesso em: 18 ago. 2016.

Guijit, I. 2008. Seeking surprise: rethinking monitoring for collective learning in rural resource management. Tese de Doutorado, Wageningen University, Holanda. Disponível em: http://ibrary.wur.nl/WebQuery/wda/ abstract/1865671. Acesso em: 20 nov. 2016.
Jorgensen, E. T., M. K. Poulsen, J. F. Lund, e J. F. Massao. 2005. Community-based monitoring of natural resource useand forest quality in montane forests and miombo woodlands of Tanzania. Biodiversity \& Conservation 14(11):2653-2677. DOI: https://doi.org/10.1007/s10531-005-8399-5.

Lawrence, A., K. Paudel, R. Barnes, e Y. Malla. 2007. Adaptive value of participatory biodiversity monitoring in community forestry, Nepal. Environmental Conservation 33(4):325-334. DOI: https://doi.org/10.1017/S0376892906003432.

Lee, W., M. Mcglone, e E. Wright. 2005. Biodiversity inventory monitoring: a review of national and international systems and a proposed framework for future biodiversity monitoring by the Department of Conservation. Nova Zelândia: Landcare Research New Zealand Ltd. Disponível em: https://tinyurl. com/y3p47orf. Acesso em: 20 out. 2016.

Magnusson, W., R. Braga Neto, F. Pezzini, F. Baccaro, H. Bergallo, J.Penha, D. Rodrigues, L. M. Verdade, A. Lima, A. L. Albernaz, J.-M. Hero, B. Lawson, C. Castilho, D. Drucker, E. Franklin, F. Mendonça, F. Costa, G. Galdino, G. Castley, J. Zuanon, J. Vale, J. L. C. Santos, R. Luizão, R. Cintra, R. I. Barbosa, A. Lisboa, R. V. Koblitz, C. N. Cunha, e A. R. Mendes Pontes. 2013. Biodiversidade e monitoramento ambiental integrado. São Paulo: Áttema Editorial. Disponível em: https://ppbio.inpa.gov.br/ sites/default/files/Biodiversidade_e_monitoramento_ ambiental_integrado.pdf. Acesso em: 15 maio 2016.

Reed, M. S., A. J. Dougill, e T. R. Baker. 2008. Participatory indicator development: what can ecologists and local communities learn from each 
other? Ecological Applications 18(5):1253-1269. DOI: https://doi.org/10.1890/07-0519.1.

Schmeller, D. S., P. Y. Henry, R. Julliard, B. Gruber, J. Clobert, F. Dziock, S. Lengyel, P. Nowick, E. Déri, E. Budrys, T. Kull, K. Tali, B. Buch, J. Settele, C. Van Swaay, A. Kobler, V. Babij, E. Papastergiadou, e K. Henle. 2009. Advantages of volunteer-based biodiversity monitoring in Europe. Conservation Biology23(2):307-316. DOI: https://doi.org/10.1111/ j.1523-1739.2008.01125.x.

Sheil, D., e A. Lawrence. 2004. Tropical biologists, local people and conservation: new opportunities for collaboration. Trends in Ecology \& Evolution 19(1)2:634638. DOI: https://doi.org/10.1016/j.tree.2004.09.019.

Stuart-Hill, G., R. Diggle, B. Munale, J. Tagg, e D. Ward. 2005. The event book system: communitybased monitoring in Namibia. Biodiversity \& Conservation 14(11):2611-2631. DOI: https://doi. org/10.1007/s10531-005-8391-0.

Whitelaw, G., H. Vaughan, B. Craige, e D. Atkinson. 2003. Establishing the Canadian community monitoring network. Environmental Monitoring and Assessment 88(1-3):409-418. DOI: https://doi. org/10.1023/A:1025545813057. 